\title{
Potential involvement of dietary advanced glycation end products in impairment of skeletal muscle growth and muscle contractile function in mice
}

\author{
Tatsuro Egawa ${ }^{1,2 *}$, Satoshi Tsuda $^{1}$, Ayumi Goto ${ }^{1,2}$, Yoshitaka Ohno ${ }^{3}$, Shingo Yokoyama ${ }^{3}$, \\ Katsumasa Goto ${ }^{2}$ and Tatsuya Hayashi ${ }^{1}$ \\ ${ }^{1}$ Laboratory of Sports and Exercise Medicine, Graduate School of Human and Environmental Studies, \\ Kyoto University, Kyoto, 606-8501, Japan \\ ${ }^{2}$ Department of Physiology, Graduate School of Health Sciences, Toyohashi Sozo University, Toyohashi, Aichi, 440-8511, Japan \\ ${ }^{3}$ Laboratory of Physiology, School of Health Sciences, Toyohashi Sozo University, Toyohashi, Aichi, 440-8511, Japan
}

(Submitted 29 August 2016 - Final revision received 5 December 2016 - Accepted 14 December 2016 - First published online 17 January 2017)

\section{Abstract}

Diets enriched with advanced glycation end products (AGE) have recently been related to muscle dysfunction processes. However, it remains unclear whether long-term exposure to an AGE-enriched diet impacts physiological characteristics of skeletal muscles. Therefore, we explored the differences in skeletal muscle mass, contractile function and molecular responses between mice receiving a diet high in AGE (H-AGE) and low in AGE (L-AGE) for 16 weeks. There were no significant differences between L-AGE and H-AGE mice with regard to body weight, food intake or epididymal fat pad weight. However, extensor digitorum longus (EDL) and plantaris (PLA) muscle weights in H-AGE mice were lower compared with L-AGE mice. Higher levels of $N^{\varepsilon}$-(carboxymethyl)-L-lysine, a marker for AGE, in EDL muscles of H-AGE mice were observed compared with L-AGE mice. H-AGE mice showed lower muscle strength and endurance in vivo and lower muscle force production of PLA muscle in vitro. mRNA expression levels of myogenic factors including myogenic factor 5 and myogenic differentiation in EDL muscle were lower in H-AGE mice compared with L-AGE mice. The phosphorylation status of 70-kDa ribosomal protein S6 kinase Thr ${ }^{389}$, an indicator of protein synthesis signalling, was lower in EDL muscle of H-AGE mice than that of L-AGE mice. These findings suggest that long-term exposure to an AGE-enriched diet impairs skeletal muscle growth and muscle contractile function, and that these muscle dysfunctions may be attributed to the inhibition of myogenic potential and protein synthesis.

\section{Key words: Muscle strength: Muscle fatigue resistance: Muscle force production: Myogenesis: Protein synthesis}

Skeletal muscles, which account for approximately $40 \%$ of total body mass, are the largest tissue in the body and play a crucial role in locomotion and metabolism. Both skeletal muscle mass and function are regulated in response to changes in nutrition, physical activity, environment and pathological conditions. Maintenance of muscle mass and function is an important public health issue, as low muscle quality is associated with clinical problems including poor glycaemic control, limited mobility and even mortality ${ }^{(1,2)}$.

Advanced glycation end products (AGE), which are produced by a non-enzymatic reaction between glucose and proteins, are closely involved in the pathogenesis of diabetes ${ }^{(3,4)}$, Alzheimer disease $^{(5)}$, cancer $^{(6)}$ and hypertension ${ }^{(7)}$. Moreover, elevated AGE in blood or skin are related to low muscle mass, grip strength and walking speed in elderly and diabetic patients ${ }^{(8-11)}$. Thus, a negative relationship between AGE and skeletal muscle function has been suggested.
AGE are endogenously formed but are also absorbed from exogenous sources such as foods, especially when prepared at elevated temperatures ${ }^{(12)}$. As dietary AGE are poorly absorbed (approximately $10 \%)^{(13)}$, their potential action in human health has gone largely unnoticed. However, recent systematic reviews of randomised-controlled trials have shown that exposure to AGE-enriched diets may be responsible for the development of chronic diseases such as diabetes, CVD and chronic kidney disease through increased inflammation and oxidative stress ${ }^{(14)}$, and that dietary restriction of AGE alleviates insulin resistance, oxidative stress and endothelial dysfunction $^{(15)}$. In addition, an AGE-enriched diet induced insulin resistance in mouse skeletal muscle ${ }^{(16)}$. Thus, we hypothesised that dietary AGE might impair skeletal muscle function. However, whether dietary AGE affect physiological characteristics of skeletal muscle is not yet fully understood. Therefore, our aim was to evaluate the effect of long-term

Abbreviations: AGE, advanced glycation end products; CML, $N^{\varepsilon}$-(carboxymethyl)-L-lysine; EDL, extensor digitorum longus; H-AGE, high in AGE; L-AGE, low in AGE; LC3, microtubule-associated protein light-chain 3; p70S6K, 70-kDa ribosomal protein S6 kinase; Pax7, paired box 7; PLA, plantaris; MHC, myosin heavy chain; mTOR, mammalian target of rapamycin; Myf5, myogenic factor 5; MyoD, myogenic differentiation; SOL, soleus.

* Corresponding author: T. Egawa, fax: +81 75753 6885, email tatsuro.egawa@gmail.com 
exposure to an AGE-enriched diet on skeletal muscle mass and contractile functions.

Myogenesis plays an important role in postnatal growth and skeletal muscle development. Sequential activation and expression of paired box 7 (Pax7) and myogenic regulatory factors including myogenic factor 5 (Myf5), myogenic differentiation (MyoD) and myogenin are essential for the initiation and progression of myogenesis ${ }^{(17)}$. An in vitro study revealed that AGE treatment for $48 \mathrm{~h}$ suppressed myogenin expression in $\mathrm{C} 2 \mathrm{C} 12$ myotubes $^{(18)}$. Further, an in vivo study showed that long-term intake of a high-fructose diet decreased MyoD expression in mouse skeletal muscle, which was attenuated by supplementation with pyridoxamine, an AGE formation inhibitor ${ }^{(19)}$. The receptor for AGE also regulates muscle differentiation through Pax 7 and myogenin ${ }^{(20)}$. These studies suggest that AGE impact the expressions of myogenic factors and inhibit myogenesis.

Skeletal muscle growth occurs when the rate of protein synthesis exceeds that of degradation. A central signalling pathway regulating protein synthesis is the Akt/mammalian target of rapamycin (mTOR)/70-kDa ribosomal protein $\mathrm{S} 6$ kinase (p70S6K) signalling pathway ${ }^{(21)}$. On the other hand, protein degradation is regulated by two major systems: autophagy and ubiquitin-proteasome systems ${ }^{(22,23)}$. Previously, AGE treatment was reported to induce autophagy by suppressing the Akt/mTOR pathway in neonatal cardiomyocytes ${ }^{(24)}$. In addition, a recent study demonstrated that AGE treatment decreased the phosphorylation status of Akt at $\operatorname{Ser}^{473}$, which is an indicator of Akt activity, and increased atrogin-1, which is a key enzyme in the ubiquitin-proteasome system, in $\mathrm{C} 2 \mathrm{C} 12$ myotubes $^{(18)}$. These findings suggest that AGE inhibit protein synthesis as well as promote protein degradation in skeletal muscle. Therefore, our secondary aim was to evaluate the influence of dietary $\mathrm{AGE}$ on the regulatory systems of myogenesis and protein turnover in skeletal muscle.

We examined the differences in muscle mass, contractile properties and molecular responses between mice that received a diet high in AGE (H-AGE) $v$. one low in AGE (L-AGE) for 16 weeks. We found that $N^{\varepsilon}$-(carboxymethyl)-L-lysine (CML), a well-characterised compound that serves as a marker for $\mathrm{AGE}^{(25)}$, accumulated in fast-type extensor digitorum longus (EDL) muscle, and EDL muscle weight was lower in H-AGE mice compared with L-AGE mice. The contractile properties were also poorer in H-AGE mice. Furthermore, mRNA expressions of myogenic factors and phosphorylation status of mTOR/p70S6K signalling pathway were lower in EDL muscles of H-AGE mice compared with L-AGE mice.

\section{Methods}

\section{Animals and treatment}

Male ICR mice (5 weeks old) were purchased from Shimizu Breeding Laboratories (Kyoto, Japan). Mice were placed in a room maintained at $22-24^{\circ} \mathrm{C}$ with a $12 \mathrm{~h}$ light $-12 \mathrm{~h}$ dark cycle and fed a standard diet (AIN-93G; Oriental Yeast) and water ad libitum. After 1 week of adjustment, mice were randomised to receive a diet either low in AGE (AIN-93G unbaked, $n$ 10) or high in AGE (AIN-93G baked for $1 \mathrm{~h}$ at $160^{\circ} \mathrm{C}, n$ 10) consistent with previous studies ${ }^{(26-28)}$. The H-AGE diet contained five times as much AGE compared with the L-AGE diet without changes in nutrient composition ${ }^{(28)}$. Both groups were followed-up for 16 weeks. Body weight and food intake were measured every week and every 2 weeks, respectively. At the end of the study period, animals were fasted for $4 \mathrm{~h}$ and anaesthetised intraperitoneally with mixtures of medetomidine hydrochloride $(0.3 \mathrm{mg} / \mathrm{kg})$, midazolam $(4.0 \mathrm{mg} / \mathrm{kg})$ and butorphanol $(5.0 \mathrm{mg} / \mathrm{kg})$. Under anaesthesia, soleus (SOL), EDL and plantaris (PLA) muscles, tibial, epididymal fat pads and blood samples were collected. All animal protocols were carried out in accordance with the Guide for the Care and Use of Laboratory Animals by the National Institutes of Health (Bethesda, MD, USA) and were approved by the Kyoto University Graduate School of Human and Environmental Studies.

\section{Grip strength test}

The grip strength test was performed $5 \mathrm{~d}$ before the end of the 16-week study. Grip strength was measured using a force transducer (DS2-50N; IMADA) according to the published protocol (DMD_M.2.2.001). In brief, mice were allowed to rest on a horizontal mesh with their forelimbs and hindlimbs (four paws) and were then gently pulled back until their grip was broken. Three consecutive measurements were obtained within $1 \mathrm{~min}$, and were averaged to determine mean grip strength. Data were normalised to body weight and expressed as N/g.

\section{Wire-hanging test}

To evaluate muscle fatigue resistance, the wire-hanging test was carried out $4 \mathrm{~d}$ before the end of the 16-week study consistent with the published protocol ${ }^{(29)}$. In brief, mice were suspended by their forelimbs from a metallic wire (2-mm thick, 50-cm long) suspended $40 \mathrm{~cm}$ above soft ground. As soon as the mice were suspended, the timer was started. The timer was stopped when the animal fell and re-started after it was placed again on the wire. The number of falls from the wire was recorded, and the test was stopped after ten falls or $180 \mathrm{~s}$ of suspension. Data were expressed as the average fall score: each animal was given a score of 10 at the beginning of the test, which was reduced by 1 after each fall. The average fall score was calculated at a given time during the test as $(10 n-x) / n$, where $n$ is the number of animals and $x$ the cumulative number of falls. The longest holding time was also recorded, and the holding impulse was calculated as $s \times g$, where $s$ is the longest holding time and $g$ the body weight.

\section{In vitro muscle contraction}

In vitro muscle contraction was analysed as described previously $^{(30,31)}$. Isolated PLA muscles were mounted on an incubation apparatus with tension set to $0.5 \mathrm{~g}$ and rested in Krebs-Ringer bicarbonate buffer (117-mm NaCl, 4.7-m $\mathrm{KCl}, 2 \cdot 5-\mathrm{mm} \mathrm{CaCl}_{2}$, 1.2-m $\mathrm{KH}_{2} \mathrm{PO}_{4}, 1 \cdot 2-\mathrm{mm} \mathrm{MgSO}_{4}, 24 \cdot 6-\mathrm{mm} \mathrm{NaHCO}_{3}$ ) containing 2-mm pyruvate for $30 \mathrm{~min}$. All buffers were continuously bubbled with $95 \% \quad \mathrm{O}_{2}-5 \% \quad \mathrm{CO}_{2}$ and maintained at $37^{\circ} \mathrm{C}$. For measuring force production, muscles were stimulated using 
an electric stimulator (SEN-3401; Nihon Kohden) at frequencies of 5, 10, 25, 50, 75 and $100 \mathrm{~Hz}$ (train duration: $1 \mathrm{~s}$; pulse duration: $0.1 \mathrm{~ms}$; voltage: $10 \mathrm{~V}$ ) with a 2 -min rest period between contractions $^{(32)}$. Subsequently, for measuring muscle fatigability, muscles were stimulated at $100 \mathrm{~Hz}, 1-\mathrm{s}$ on 1-s off, for $30 \mathrm{~s}$. Force was monitored using a force transducer (TRN001; Kent Scientific) and recorded (U-228-2P-500; Pantos). Force production data were normalised to muscle weight and expressed as $\mathrm{N} / \mathrm{g}$.

\section{Real-time RT-PCR analyses}

Real-time RT-PCR analyses were performed as previously described $^{(33)}$. In brief, total muscle RNA was extracted using a miRNeasy Mini kit (Qiagen) according to the manufacturer's protocol. RNA was reverse-transcribed to complementary DNA (cDNA) using PrimeScript RT Master Mix (Takara Bio); synthesised cDNA was then subjected to real-time RT-PCR (StepOne, Applied Biosystems) using Takara SYBR Premix Ex Taq II (Takara Bio). The relative fold change of expression was calculated by the comparative CT method using StepOne software version 2.3. To normalise the amount of total RNA present in each reaction, glyceraldehyde 3-phosphate dehydrogenase was used as an internal standard. The primer sequences are shown in Table 1.

\section{Measurement of myosin heavy chain isoform composition}

Analysis of myosin heavy chain (MHC) isoform composition (I, IIa, IIx and IIb) was carried out using a modification of previously described methods ${ }^{(34)}$. In brief, muscles were homogenised in ice-cold lysis buffer (1:40, w/v) containing 20-mu Tris· $\mathrm{HCl}(\mathrm{pH} 7 \cdot 4)$, $1 \%$ Triton X, 50-mm NaCl, 250-mm sucrose, 50-mм NaF, 5-mм sodium pyrophosphate, 2-mm dithiothreitol, 4-mg/1 leupeptin, 50-mg/l trypsin inhibitor, 0.1-mm benzamidine and 0.5-mm phenylmethylsulphonyl fluoride (buffer A) and centrifuged at $16000 \mathrm{~g}$ for $40 \mathrm{~min}$ at $4^{\circ} \mathrm{C}$. Extracted proteins were solubilised in Laemmli sample buffer and boiled for $5 \mathrm{~min}$. The sample proteins $(5 \mu \mathrm{g})$ were separated on SDS-polyacrylamide (7\%) gel at $120 \mathrm{~V}$ for $19 \mathrm{~h}$ in a temperature-controlled chamber at $4^{\circ} \mathrm{C}$.

Table 1. List of primer sequences (forward, reverse) used for real-time RT-PCR analyses

\begin{tabular}{ll}
\hline Protein (gene) & Sequences $\left(5^{\prime} \rightarrow 3^{\prime}\right)$ \\
\hline Pax7 (Pax7) & ATTAGCCGAGTGCTCAGAATCAA \\
& GAGGTCGGGTTCTGATTCCA \\
Myf5 (Myf5) & CTATTACAGCCTGCCGGGACA \\
MyoD (Myod1) & GACAAGCAATCCAAGCTGGACA \\
& TCATAGAAGTCGTCTGCTGTCTCAA \\
Myogenin (Myogenin) & CAGTGAATGCAACTCCCACAG \\
MuRF1 (Trim63) & TGGACGTAAGGGAGTGCAGA \\
Atrogin-1 (Fbxo32) & TTCTCGTCCAGGATGGCGTA \\
GAPDH (Gapdh) & TGTCCTTGAATTCAGCAAGCAAAC \\
& TGTGGCCATCCATTATTTCCAG \\
& TGTGTCCGTCGTGGATCTGA \\
\hline
\end{tabular}

Pax7, paired box 7; Myf5, myogenic factor 5; MyoD, myogenic differentiation; MuRF1, muscle RING finger 1; GAPDH, glyceraldehyde 3-phosphate dehydrogenase.
After electrophoresis, the gels were stained with Oriole ${ }^{\mathrm{TM}}$ Fluorescent Gel Stain (Bio-Rad Laboratories). The gels were visualised using ImageCapture G3 (Liponics) and analysed using Image J software (National Institutes of Health). As we could not clearly distinguish between the IIa and the IIx MHC bands, this phenotype was expressed as $\mathrm{IIa} / \mathrm{x}$.

\section{Western blot analyses}

Western blot analysis was performed as described previously ${ }^{(35,36)}$. Extracted samples in buffer A were solubilised in Laemmli's sample buffer and boiled for $5 \mathrm{~min}$. The samples (10 $\mu \mathrm{g}$ of protein) were separated by SDS-PAGE using $10 \%$ polyacrylamide gel at a constant current of $40 \mathrm{~mA} / \mathrm{gel}$ for $90 \mathrm{~min}$. After SDS-PAGE, the proteins were transferred to polyvinylidene fluoride membranes (Hybond-P; GE Healthcare) by using trans-blot cell (Bio-Rad) at a constant voltage of $100 \mathrm{~V}$ for $1 \mathrm{~h}$ at $4^{\circ} \mathrm{C}$. After the transfer, the membranes were blocked for $1 \mathrm{~h}$ using fish gelatin (Takara Bio). Next, the membranes were incubated overnight at $4^{\circ} \mathrm{C}$ with primary antibodies: Akt (9272; Cell Signaling Technology), Akt Ser ${ }^{473}$ (9271; Cell Signaling Technology), p70S6K (2708; Cell Signaling Technology), p70S6K Thr ${ }^{389}$ (9206; Cell Signaling Technology) and microtubule-associated protein light-chain 3 (LC3) (2775; Cell Signaling Technology). The membranes were washed with Tris-buffered saline with $0 \cdot 1 \%$ Tween 20 (TBS-T, pH 7.5) and then allowed to react with anti-rabbit IgG (Cell Signaling Technology) for $1 \mathrm{~h}$ at room temperature. After the final wash with TBS-T, protein bands were visualised using chemiluminescence (Wako). Signal density was measured using Light-Capture (AE-6971; ATTO Corporation).

\section{Measurement of $\mathrm{N}^{\varepsilon}$-(carboxymethyl)-L-lysine content}

The muscle sample was homogenised in buffer $\mathrm{A}$, and the supernatant was collected after centrifugation at $16000 \mathrm{~g}$ for $40 \mathrm{~min}$ at $4^{\circ} \mathrm{C}$. Blood samples were collected from the carotid artery into tubes containing heparin as anticoagulant, and plasma was separated by centrifugation at $8000 \mathrm{~g}$ for $15 \mathrm{~min}$. The content of CML in muscles and plasma was measured using a CircuLex $\mathrm{CML} / N^{E}$-(carboxymethyl) lysine ELISA Kit (Medical \& Biological Laboratories) according to the manufacturer's protocol. Protein concentration of the supernatant of muscles and plasma was determined using the Bradford technique, and the CML content in samples was expressed as $\mu \mathrm{g} / \mathrm{mg}$ protein.

\section{Statistical analyses}

Values are expressed as means with their standard errors. Statistical significance was analysed using parametric Student's $t$ test or non-parametric Mann-Whitney $U$ test (Tables 2-4 and Fig. 1) and two-way ANOVA with diet and frequencies (Fig. 2) or diet and muscles (Fig. 3-5) as main factors. In the event of significant main effects and/or interactions, post hoc TukeyKramer tests were performed. Differences between groups were considered statistically significant at $P<0.05$. The sample size was calculated by power analysis at 0.8 and a level of significance of 0.05 with a hypothesised effect size of 1.2 based on the means and variances of muscle mass data in our 
previous study ${ }^{(37)}$, which estimated the sample size to be 10 . All statistical analyses were performed using Ekuseru-Toukei 2012 software (Social Survey Research Information)

\section{Results}

\section{Dietary advanced glycation end products suppressed} skeletal muscle growth

The characteristics of mice exposed to L-AGE and H-AGE are shown in Table 2 . There were no significant differences between L-AGE and H-AGE mice in body weight, tibial length, food intake and epididymal fat pad weight. EDL muscle weight was lower in H-AGE mice compared with L-AGE mice when normalised to body weight and tibial length. PLA muscle weight was lower in H-AGE mice when normalised to tibial length and tended to be lower when normalised to body weight $(P=0 \cdot 096)$. There were no significant differences in SOL muscle weight between L-AGE and H-AGE mice.

\section{Dietary advanced glycation end products increased $\mathrm{N}^{\varepsilon}$ -} (carboxymethyl)-L-lysine accumulation in skeletal muscle

To evaluate the impact of the H-AGE diet for 16 weeks, we examined accumulation of CML in skeletal muscles and plasma. H-AGE mice showed an increase in CML content in EDL muscle (Table 3). The content in SOL muscle and plasma tended to increase, but this was not significantly different.

\section{Dietary advanced glycation end products deteriorated}

\section{muscle performance in vivo}

To examine the effect of the H-AGE diet on muscle performance, we assessed muscle strength and fatigue resistance in mice. The grip strength test was used to measure muscle strength. Grip strength in all four limbs in H-AGE mice was weaker than in L-AGE mice (Fig. 1(a)). To measure fatigue

Table 2. Characteristics of low in advanced glycation end products (L-AGE)- and high in AGE (H-AGE)-fed mice

(Mean values with their standard errors; $n 10$ per group)

\begin{tabular}{|c|c|c|c|c|}
\hline & \multicolumn{2}{|c|}{ L-AGE } & \multicolumn{2}{|c|}{ H-AGE } \\
\hline & Mean & SE & Mean & SE \\
\hline Initial body weight (g) & 31.5 & 0.8 & 31.5 & 0.8 \\
\hline Final body weight $(\mathrm{g})$ & $53 \cdot 6$ & 2.5 & $59 \cdot 7$ & 4.0 \\
\hline Tibial length $(\mathrm{cm})$ & $2 \cdot 01$ & 0.02 & 2.06 & 0.02 \\
\hline Food intake (kJ/d) & $79 \cdot 1$ & 1.7 & $78 \cdot 2$ & 1.7 \\
\hline Food intake (kcal/d) & 18.9 & 0.4 & $18 \cdot 7$ & 0.4 \\
\hline \multicolumn{5}{|c|}{ Tissue weight normalised to tibial length $(\mathrm{mg} / \mathrm{cm})$} \\
\hline SOL & 5.8 & 0.2 & 5.5 & 0.2 \\
\hline EDL & $7 \cdot 8$ & 0.3 & $7 \cdot 0^{\star}$ & 0.2 \\
\hline PLA & $12 \cdot 3$ & 0.3 & $11.4^{\star}$ & 0.4 \\
\hline Epididymal fat pad weight & $1077 \cdot 1$ & 137.4 & $1215 \cdot 3$ & $136 \cdot 4$ \\
\hline \multicolumn{5}{|c|}{ Tissue weight normalised to body weight $(\mathrm{mg} / \mathrm{g})$} \\
\hline SOL & 0.22 & 0.01 & 0.20 & 0.01 \\
\hline EDL & 0.30 & 0.02 & $0.25^{*}$ & 0.02 \\
\hline PLA & 0.46 & 0.01 & 0.41 & 0.03 \\
\hline Epididymal fat pad weight & 39.7 & 4.4 & 41.6 & 3.84 \\
\hline
\end{tabular}

$\mathrm{SOL}$, soleus; EDL, extensor digitorum longus; PLA, plantaris. ${ }^{\star} P<0.05 v$. L-AGE.
Table 3. $N^{E}$-(carboxymethyl)-L-lysine content (Mean values with their standard errors; $n$ 8-10 per group)

\begin{tabular}{|c|c|c|c|c|}
\hline & \multicolumn{2}{|c|}{ L-AGE ( $\mu \mathrm{g} / \mathrm{mg}$ protein) } & \multicolumn{2}{|c|}{ H-AGE ( $\mu \mathrm{g} / \mathrm{mg}$ protein) } \\
\hline & Mean & $\mathrm{SE}$ & Mean & SE \\
\hline SOL & 0.18 & 0.01 & 0.21 & 0.01 \\
\hline EDL & 0.20 & 0.01 & $0.28^{\star}$ & 0.02 \\
\hline Plasma & 0.022 & 0.006 & 0.033 & 0.005 \\
\hline
\end{tabular}

L-AGE, low in advanced glycation end products; H-AGE, high in advanced glycation end products; SOL, soleus; EDL, extensor digitorum longus; PLA, plantaris. ${ }^{*} P<0.05 v$. L-AGE.

resistance, we carried out the wire-hanging test. The average fall score during the wire-hanging test was 3.7 at the end of the experiment in L-AGE mice, whereas the score fell faster in H-AGE mice and reached 1.4 (Fig. 1(b)). The longest holding time during the wire-hanging test was shorter by $30 \%$ in H-AGE mice compared with L-AGE mice; holding impulse was significantly lower in H-AGE mice than in L-AGE mice (Fig. 1(c)).

\section{Dietary advanced glycation end products deteriorated muscle force production in vitro}

We next measured force production in skeletal muscle in vitro to determine the effect of $\mathrm{H}-\mathrm{AGE}$ on muscle contractile property. PLA muscle of H-AGE mice was found to produce significantly lower force at 75 and $100 \mathrm{~Hz}$ compared with L-AGE mice (Fig. 2(a)). There were no significant differences in the patterns of force decline between L-AGE and H-AGE mice (Fig. 2(b)).

\section{Dietary advanced glycation end products did not affect myosin heavy chain isoform composition in skeletal muscle}

As muscle fibre type composition is a determinant of muscle strength and fatigue resistance, we evaluated the effect of H-AGE on MHC isoform composition in skeletal muscles. No significant differences were observed in MHC isoform composition in either SOL or EDL muscles between L-AGE and H-AGE mice (Table 4)

\section{Dietary advanced glycation end products decreased mRNA expressions of myogenic factors in skeletal muscle}

To determine the effect of H-AGE on regulatory factors related to skeletal muscle growth, we evaluated the expression levels of mRNA involved in myogenesis. Myf5 mRNA expression level was significantly decreased in EDL muscle of H-AGE mice (Fig. 3). In addition, MyoD mRNA expression levels were lower in H-AGE mice compared with L-AGE mice. Pax7 and myogenin mRNA expression levels did not differ significantly based on an H-AGE diet.

\section{Dietary advanced glycation end products suppressed protein synthesis signalling in skeletal muscle}

To examine the effect of H-AGE on protein synthesis, we evaluated the phosphorylation status of Akt/mTOR/p70S6K 
(a)

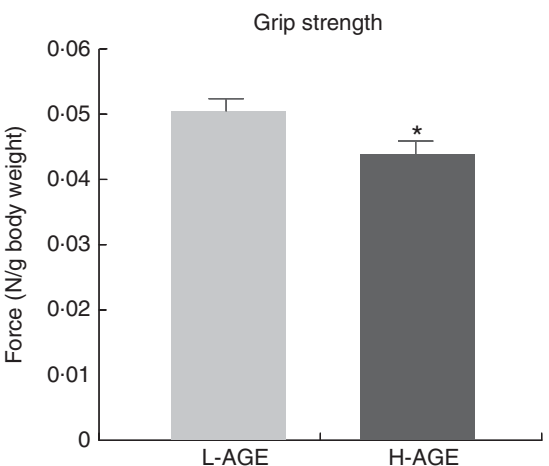

(b)
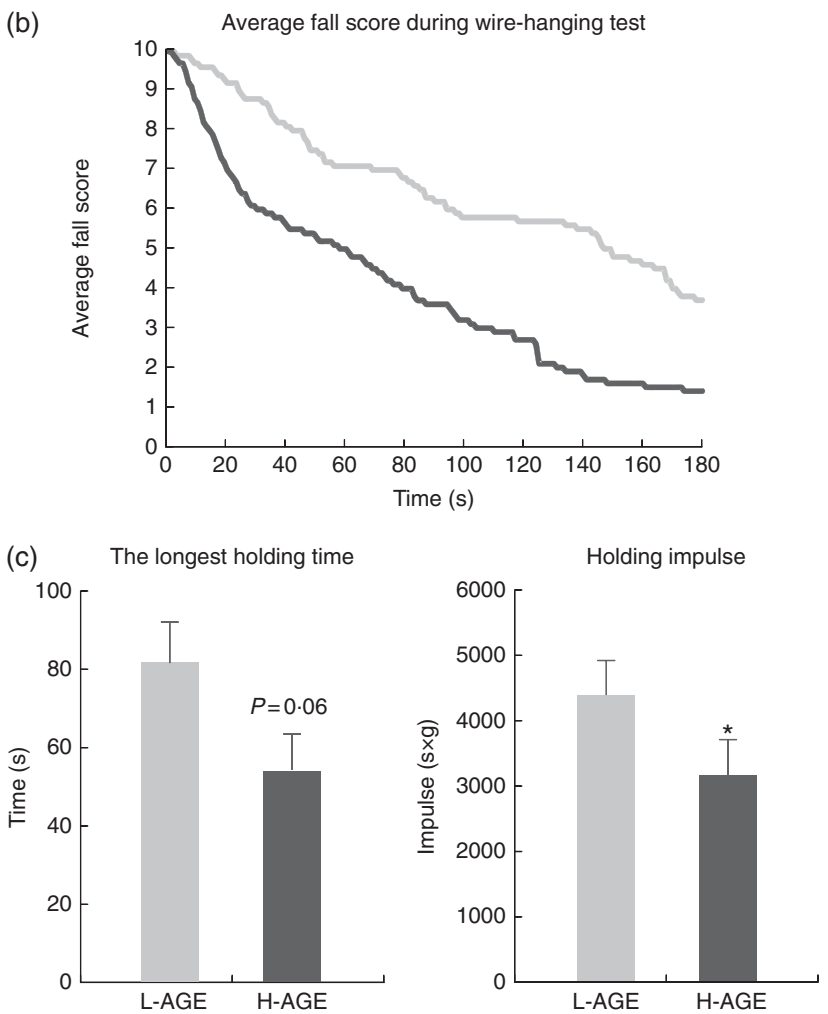

Fig. 1. The grip strength test and wire-hanging test in mice fed a diet low in advanced glycation end products (L-AGE) or a diet high in AGE (H-AGE). (a) Forelimb and hindlimb (four paws) grip strength. (b) Average fall score during the wire-hanging test. (c) Longest holding time and holding impulse during the wire-hanging test. The grip strength test and wire-hanging test were carried out 5 and $4 \mathrm{~d}$ before the end of the 16-week study, respectively. Values are means ( $n 10$ per group), with their standard errors. ${ }^{*} P<0.05 \mathrm{v}$. L-AGE mice. $\_$, L-AGE; — $\longrightarrow$ H-AGE.

signalling pathway. The phosphorylation status of Akt at $\operatorname{Ser}^{473}$ was not affected by the H-AGE diet, but the expression of phosphorylated $\mathrm{p} 70 \mathrm{~S} 6 \mathrm{~K} \mathrm{Thr}^{389}$, an indicator of mTOR activity, was lower in H-AGE mice compared with L-AGE mice (Fig. 4).

Dietary advanced glycation end products had no effect on protein degradation pathways in skeletal muscle

To analyse the effect of H-AGE on protein degradation, we evaluated the expression levels of molecules involved in
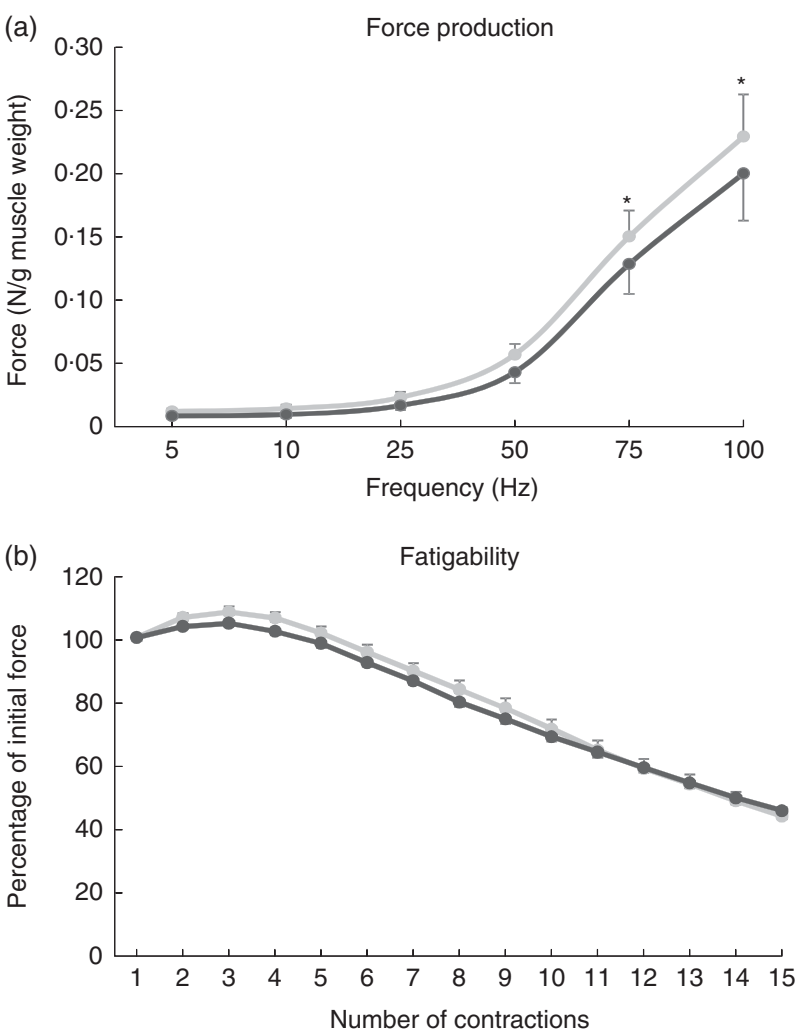

Fig. 2. In vitro force production and fatigability of plantaris muscles in L-AGE or H-AGE mice. (a) Force generation in response to electrical stimulation. (b) Percentage of force drop during fifteen contraction cycles. Isolated plantaris muscle was allowed to rest for $30 \mathrm{~min}$, and the muscle was tetanically contracted at frequencies of $0,5,10,25,50,75$ and $100 \mathrm{~Hz}$ with a 2-min rest between contractions. A fatigue run was then carried out by stimulating the muscle at $100 \mathrm{~Hz}, 1-\mathrm{s}$ on $1-\mathrm{s}$ off, for $30 \mathrm{~s}$. Values are means ( $n 10$ per group), with their standard errors. ${ }^{\star} P<0.05 v$. L-AGE mice. L-AGE, low in advanced glycation end products; H-AGE, high in AGE; — , L-AGE; — , H-AGE.

Table 4. Myosin heavy chain (MHC) isoform composition (Mean values with their standard errors; $n 10$ per group)

\begin{tabular}{|c|c|c|c|c|}
\hline & \multicolumn{2}{|c|}{ L-AGE } & \multicolumn{2}{|c|}{ H-AGE } \\
\hline & Mean & SE & Mean & SE \\
\hline \multicolumn{5}{|l|}{ SOL } \\
\hline MHC I (\%) & $62 \cdot 0$ & $2 \cdot 0$ & $60 \cdot 3$ & 1.5 \\
\hline $\mathrm{MHC} \| \mathrm{la} / \mathrm{x}(\%)$ & $38 \cdot 0$ & $2 \cdot 0$ & $39 \cdot 7$ & 1.5 \\
\hline MHC IIb (\%) & \multicolumn{2}{|c|}{ ND } & \multicolumn{2}{|c|}{ ND } \\
\hline \multicolumn{5}{|l|}{ EDL } \\
\hline MHC I (\%) & \multicolumn{2}{|c|}{ ND } & \multicolumn{2}{|c|}{ ND } \\
\hline $\mathrm{MHC} \| \mathrm{la} / \mathrm{x}(\%)$ & 32.7 & 0.9 & 33.6 & 1.4 \\
\hline MHC IIb (\%) & $67 \cdot 3$ & 0.9 & $66 \cdot 4$ & 1.4 \\
\hline
\end{tabular}

L-AGE, low in advanced glycation end products; H-AGE, high in advanced glycation end products; SOL, soleus; EDL, extensor digitorum longus.

autophagy and ubiquitin-proteasome systems. The relative expression levels of LC3II/LC3I, an indicator of autophagy activity, were not affected by the H-AGE diet (Fig. 5). There were also no differences between L-AGE and H-AGE mice with regard to mRNA expressions of atrogin-1 and muscle RING finger 1 (MuRF1), another key enzyme of the ubiquitinproteasome system (Fig. 5). 


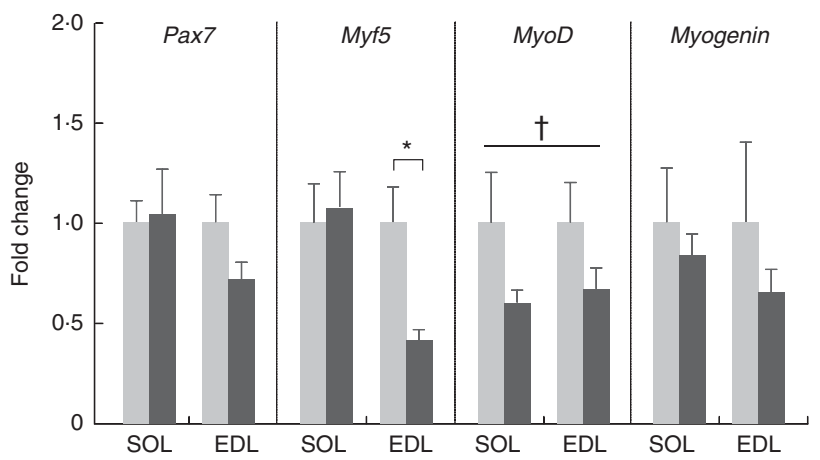

Fig. 3. Relative expressions of mRNA specific for myogenesis in L-AGE or $\mathrm{H}$-AGE mice. Soleus (SOL) and extensor digitorum longus (EDL) muscles were dissected, and expressions of paired box 7 (Pax7), myogenic factor 5 (Myf5), myogenic differentiation (MyoD) and myogenin were measured using real-time RT-PCR analyses. Values are means ( $n 10$ per group), with their standard errors. ${ }^{*} P<0.05 v$. L-AGE mice. †Significant main effect of diet (L-AGE $v$. $\mathrm{H}$-AGE mice). L-AGE, low in advanced glycation end products; H-AGE, high in AGE; $\square$, L-AGE; $\square$, H-AGE.
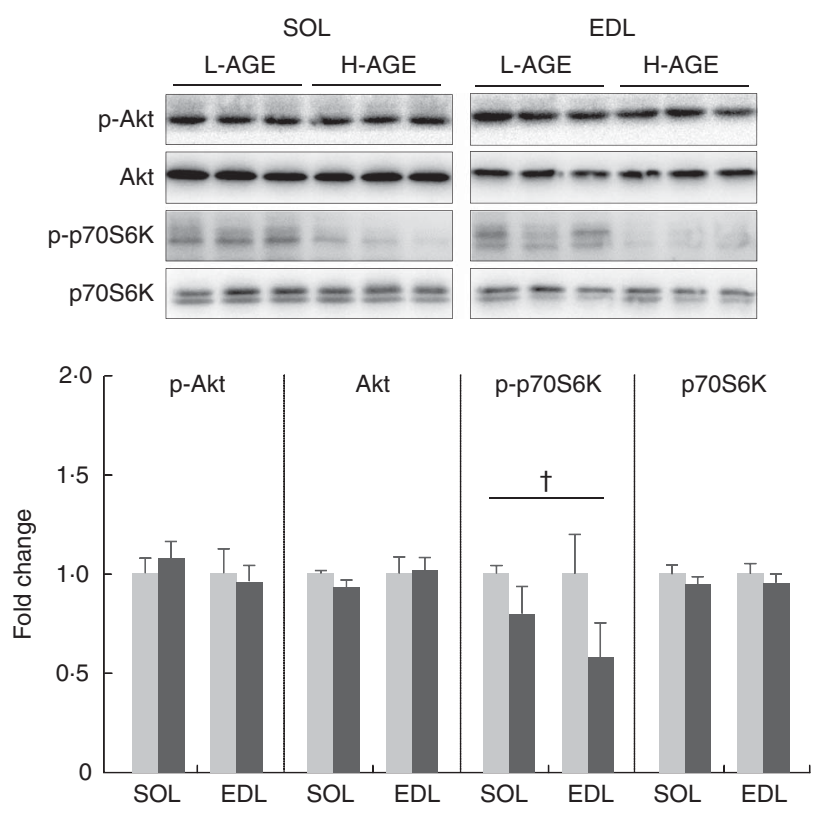

Fig. 4. Relative expressions of protein specific for protein synthesis pathway in L-AGE or H-AGE mice. Soleus (SOL) and extensor digitorum longus (EDL) muscles were dissected, and expressions of Akt and phosphorylated Akt $\mathrm{Ser}^{473}$ (p-Akt), 70-kDa ribosomal protein $\mathrm{S} 6$ kinase (p70S6K) and phosphorylated p70S6K Thr ${ }^{389}$ (p-p70S6K) were measured using western blot analyses. Representative immunoblots are also shown. Values are means (n 10 per group), with their standard errors. †Significant main effect of diet (L-AGE v. H-AGE mice). L-AGE, low in advanced glycation end products; $\mathrm{H}-\mathrm{AGE}$, high in AGE; $\square$, L-AGE; $\square$, H-AGE.

\section{Discussion}

The results of the present study revealed several novel findings about the effects of dietary AGE on skeletal muscle functions. First, mice fed an H-AGE diet for 16 weeks exhibited smaller muscle mass than L-AGE mice, which was accompanied by CML accumulation in skeletal muscle. Second, exposure to H-AGE induced skeletal muscle dysfunction, including muscle strength, fatigue resistance and in vitro muscle force
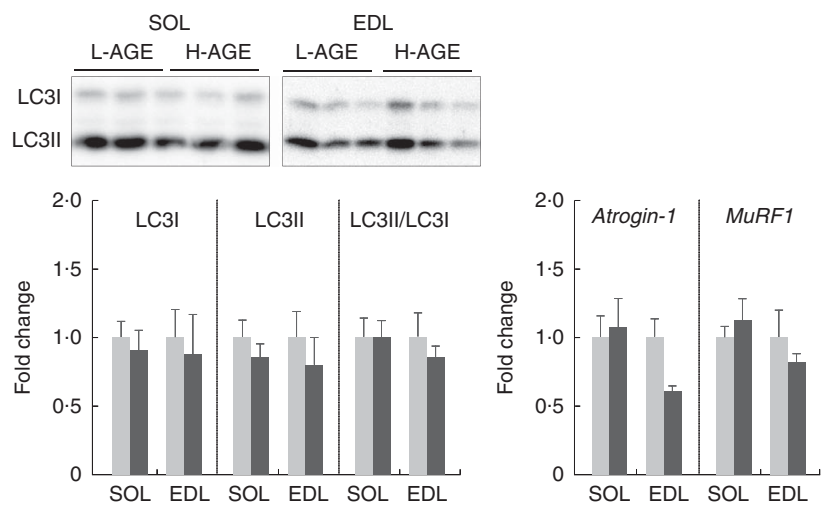

Fig. 5. Relative expressions of protein and mRNA specific for protein degradation pathway in L-AGE or H-AGE mice. Soleus (SOL) and extensor digitorum longus (EDL) muscles were dissected, and protein expressions of microtubule-associated protein light-chain 3 (LC3)-I and II were measured using western blot analyses, and mRNA expressions of atrogin-1 and muscle RING finger 1 (MuRF1) were measured using real-time RT-PCR analyses. Representative immunoblots are also shown. Values are means ( $n 10$ per group), with their standard errors. L-AGE, low in advanced glycation end products; H-AGE, high in AGE; $\square$, L-AGE; $\square$, H-AGE.

production, without changes in MHC isoform composition. Third, mRNA expressions of myogenic factors, Myf5 and MyoD, in skeletal muscle were lower in H-AGE mice compared with L-AGE mice. Fourth, phosphorylation status of p70S6K Thr ${ }^{389}$ was lower in H-AGE mice than in L-AGE mice.

Recently, an epidemiological study has suggested a relationship between AGE accumulation and skeletal muscle mass in humans ${ }^{(11)}$. In addition, an experimental study showed that AGE treatment in cultured muscle cells suppressed MyoD and promoted atrophy ${ }^{(18)}$. That study also revealed that skeletal muscle atrophy in diabetic mice was attenuated by treatment with alagebrium chloride, a breaker of AGE-based crosslinks ${ }^{(18)}$. These observations indicate that AGE are potent factors that influence muscle mass. In the present study, H-AGE mice showed smaller muscle masses of EDL and PLA compared with L-AGE mice (Table 2), which was accompanied by elevated CML content (Table 3). This is the first report of long-term exposure to an AGE-enriched diet promoting AGE accumulation in skeletal muscles and impaired skeletal muscle growth.

AGE are also related to muscle dysfunction. It has been reported that elevated blood CML is associated with poor grip strength $^{(8)}$ and slow walking speed ${ }^{(9)}$. Furthermore, men with higher skin AGE levels had lower muscle strength and power $^{(10)}$. In the present study, we found that grip strength and wire-holding performance were poorer in H-AGE mice compared with L-AGE mice (Fig. 1). In addition, we showed that in vitro muscle force production normalised to muscle weight was lower in $\mathrm{H}$-AGE mice than in L-AGE mice (Fig. 2(a)). These findings indicate that dietary AGE affect muscle contractile properties in addition to muscle mass.

In this regard, some reports have shown that myofibrillar proteins are subject to glycation modification. Glycation of actomyosin increased with ageing in mice and rats $^{(38)}$. Glycation of myosin altered filament formation ${ }^{(39)}$, structure and ATPase activity ${ }^{(40-42)}$. AGE-modified actin is also accumulated with ageing ${ }^{(43)}$. Therefore, the modification of muscle 
contractile proteins such as myosin and actin is a possible mechanism inducing the loss of muscle contractile properties.

Muscle strength is also affected by muscle fibre type composition $^{(44)}$. The fibre types based on MHC isoforms essentially reflect muscle contractile properties ${ }^{(45)}$. In the present study, MHC isoform composition did not differ between L-AGE and H-AGE mice (Table 4), indicating that dietary AGE-induced muscle dysfunction is not attributed to the alteration of muscle fibre type composition.

In the present study, dietary AGE affected muscle mass more in fast-type muscles, EDL and PLA, than in slow-type muscles, SOL (Table 2). This indicates that the susceptibility to AGE might be dependent on fibre type. Indeed, CML accumulated in EDL muscle but not in SOL muscle during the 16-week treatment period (Table 3). In addition, previous reports have shown that AGE accumulation in diabetic rat skeletal muscle was greater in fast-type fibre ${ }^{(46)}$. These findings suggest that dietary AGE promote AGE accumulation preferentially in fast-type fibres of whole-body muscle, and thereby induce decreased muscle performance. However, some reports have shown that the presence of AGE did not differ between fibre types in diabetic ${ }^{(47)}$ or ageing rats ${ }^{(48)}$. Thus, additional studies are required to elucidate any dependency on fibre type for AGE influence.

Postnatal skeletal muscle growth is regulated by the sequential expressions of myogenic factors such as Pax7, Myf5, MyoD and myogenin. Pax7 is the specific marker for quiescent and activated muscle satellite cells, which are the resident skeletal muscle stem cells ${ }^{(49,50)}$. Myf5 is expressed during embryonic development and integrates various developmental signalling pathways to initiate myogenesis ${ }^{(51)}$. MyoD is considered a master control gene for $\mathrm{MyoD}^{(52)}$; myogenin participates in later stages of myogenesis and is directly associated with the differentiation process that leads to myotube formation and maturation ${ }^{53,54)}$. In the present study, Myf 5 mRNA expression level in EDL muscle was lower in H-AGE mice than in L-AGE mice (Fig. 3). Furthermore, MyoD mRNA expression level was suppressed in H-AGE mice compared with L-AGE mice. These findings suggest that dietary AGE inhibit muscle formation during a relatively early stage of myogenesis, resulting in muscle growth deterioration.

MyoD is also a crucial modulator of muscle fibre type. MyoD binds to an enhancer box (E-box) element in the MHC promoter and induces its transcription ${ }^{(55)}$. In particular, MyoD expression is high in fast-type muscle and is involved in the induction of fast-type fibre phenotype ${ }^{(56)}$. Over-expression of MyoD has been shown to up-regulate the expression of fast-type MHC ${ }^{(57)}$. Conversely, MyoD-knock-out mice showed a shift towards slower fibre phenotype in fast-type muscles ${ }^{(56,58)}$. These observations raise the possibility that the down-regulation of MyoD mRNA expression in H-AGE mice induced fibre-type transition towards a slower muscle phenotype. However, dietary AGE did not affect MHC isoform composition in the present study (Table 4). In this respect, some studies have reported inconsistent shifts in MHC with MyoD expressions ${ }^{(59,60)}$. Thus, the alteration of MyoD expression may not always be linked to the shift in MHC isoform distributions.
In addition to myogenesis, protein synthesis and subsequent accumulation of protein are necessary for skeletal muscle growth. A previous study indicated that AGE induced myotube atrophy via inhibition of the Akt pathway ${ }^{(18)}$. In the present study, the phosphorylation status of p7056K Thr ${ }^{389}$ was lower in EDL muscle of H-AGE mice compared with L-AGE mice, although no change was observed in Akt Ser ${ }^{473}$ phosphorylation (Fig. 4). These results suggest that dietary AGE affect the mTOR/p70S6K signalling pathway independently of Akt in skeletal muscle. On the other hand, AGE may possibly modulate protein degradation as noted by previous studies, which have shown that AGE treatment in cultured skeletal and cardiac muscles activated autophagy and ubiquitin-proteasome pathways $^{(18,24)}$. However, no influences on the ratio of LC3II:LC3I and mRNA expressions of atrogin-1 and MuRF1 were observed (Fig. 5), which suggests that dietary AGE do not affect protein degradation. Therefore, it is suggested that dietary AGE deteriorate skeletal muscle growth by inhibiting protein synthesis.

AGE are generally found in several foods prepared at high temperatures ${ }^{(12)}$. Therefore, animal models exposed to heattreated foods are often used to evaluate the influence of AGE in vivo ${ }^{(16,26-28,61-65)}$. However, a limitation in using these models is that the heat applied may produce unexpected products in diets. From this perspective, our study cannot exclude the possibility that products other than AGE may affect muscle dysfunction. Therefore, further investigations using inhibitors of AGE accumulation should be conducted to understand the direct links between AGE and muscle functions.

In conclusion, in the present study, we showed that exposure to an AGE-enriched diet for 16 weeks promoted CML accumulation in skeletal muscle. Furthermore, we observed impairment in skeletal muscle functions including skeletal muscle mass, muscle strength and fatigue resistance in H-AGE mice compared with L-AGE mice. AGE-enriched diet-associated skeletal muscle dysfunction might be due to a failure of postnatal myogenesis and protein synthesis. The present study shows a possibility that long-term exposure to an AGE-enriched diet impairs postnatal growth and skeletal muscle development.

\section{Acknowledgements}

We thank all the members of the Laboratory of Sports and Exercise Medicine, Kyoto University and the Laboratory of Physiology, Toyohashi SOZO University for their help. We also thank Kyoto University Research Center for Low Temperature and Materials Sciences for instrumental support.

This study was supported in part by JSPS KAKENHI grant numbers 26560371 (T. E.), 16K12942 (Y. O.), 16K16450 (S. Y.), 16K13022 (K. G.), 26350818 (K. G.) and 15K01711 (T. H.); JSPS Fellows (A. G., 14J00286); the Ministry of Agriculture, Forestry and Fisheries; Integration Research for Agriculture and Interdisciplinary Fields (funding agency: Bio-oriented Technology Research Advancement Institution, NARO) (T. H., 14532022); the Council for Science, Technology and Innovation; and SIP (funding agency: NARO) (T. H., 14533567). Additional research grants were provided by the Nakatomi Foundation (T. E.); the All Japan Coffee Association (T. E. and K. G.); the Vascular 
Disease Research Foundation (T. H.); the Naito Foundation (K. G.); the Descente Sports Foundation (K. G.); and the Graduate School of Health Sciences, Toyohashi Sozo University (K. G.).

T. E., K. G. and T. H. were involved in the conception and design of the study; T. E., S. T. and A. G. performed experiments; T. E., A. G. and S. Y. analysed the data; T. E., Y. O., S. Y., K. G. and T. H. interpreted the results of experiments; T. E. prepared the figures; T. E., S. Y. and K. G. drafted the manuscript; T. E., A. G., Y. O, K. G. and T. H. edited and revised the manuscript; T. E., S. T., A. G., Y. O., S. Y., K. G. and T. H. approved the final version of the manuscript.

The authors have no conflicts of interest to declare.

\section{References}

1. Newman AB, Kupelian V, Visser M, et al. (2006) Strength, but not muscle mass, is associated with mortality in the health, aging and body composition study cohort. J Gerontol A Biol Sci Med Sci 61, 72-77.

2. Park SW, Goodpaster BH, Strotmeyer ES, et al. (2006) Decreased muscle strength and quality in older adults with type 2 diabetes: the health, aging, and body composition study. Diabetes 55, 1813-1818.

3. Vlassara H \& Uribarri J (2014) Advanced glycation end products (AGE) and diabetes: cause, effect, or both? Curr Diab Rep 14, 453.

4. Bohlender JM, Franke S, Stein G, et al. (2005) Advanced glycation end products and the kidney. Am J Physiol Renal Physiol 289, F645-F659.

5. Takeuchi M \& Yamagishi S (2008) Possible involvement of advanced glycation end-products (AGEs) in the pathogenesis of Alzheimer's disease. Curr Pharm Design 14, 973-978.

6. van Heijst JW, Niessen HW, Hoekman K, et al. (2005) Advanced glycation end products in human cancer tissues: detection of $\mathrm{N}$ epsilon-(carboxymethyl)lysine and argpyrimidine. Ann N Y Acad Sci 1043, 725-733.

7. McNulty M, Mahmud A \& Feely J (2007) Advanced glycation end-products and arterial stiffness in hypertension. $A m J$ Hypertens 20, 242-247.

8. Dalal M, Ferrucci L, Sun K, et al. (2009) Elevated serum advanced glycation end products and poor grip strength in older community-dwelling women. J Gerontol A Biol Sci Med Sci 64, 132-137.

9. Semba RD, Bandinelli S, Sun K, et al. (2010) Relationship of an advanced glycation end product, plasma carboxymethyllysine, with slow walking speed in older adults: the InCHIANTI study. Eur J Appl Physiol 108, 191-195.

10. Momma H, Niu K, Kobayashi Y, et al. (2011) Skin advanced glycation end product accumulation and muscle strength among adult men. Eur J Appl Physiol 111, 1545-1552.

11. Kato M, Kubo A, Sugioka Y, et al. (2016) Relationship between advanced glycation end-product accumulation and low skeletal muscle mass in Japanese men and women. Geriatr Gerontol Int.

12. O'Brien J \& Morrissey PA (1989) Nutritional and toxicological aspects of the Maillard browning reaction in foods. Crit Rev Food Sci Nutr 28, 211-248.

13. Koschinsky T, He CJ, Mitsuhashi T, et al. (1997) Orally absorbed reactive glycation products (glycotoxins): an environmental risk factor in diabetic nephropathy. Proc Natl Acad Sci U S A 94, 6474-6479.

14. Clarke RE, Dordevic AL, Tan SM, et al. (2016) Dietary advanced glycation end products and risk factors for chronic disease: a systematic review of randomised controlled trials. Nutrients 8, 125.

15. Kellow NJ \& Savige GS (2013) Dietary advanced glycation end-product restriction for the attenuation of insulin resistance, oxidative stress and endothelial dysfunction: a systematic review. Eur J Clin Nutr 67, 239-248.

16. Cassese A, Esposito I, Fiory F, et al. (2008) In skeletal muscle advanced glycation end products (AGEs) inhibit insulin action and induce the formation of multimolecular complexes including the receptor for AGEs. J Biol Chem $\mathbf{2 8 3}$, 36088-36099.

17. Bentzinger CF, Wang YX \& Rudnicki MA (2012) Building muscle: molecular regulation of myogenesis. Cold Spring Harbor Perspect Biol 4, a008342.

18. Chiu CY, Yang RS, Sheu ML, et al. (2016) Advanced glycation end-products induce skeletal muscle atrophy and dysfunction in diabetic mice via a RAGE-mediated, AMPK-down-regulated, Akt pathway. J Pathol 238, 470-482.

19. Mastrocola R, Nigro D, Chiazza F, et al. (2016) Fructosederived advanced glycation end-products drive lipogenesis and skeletal muscle reprogramming via SREBP-1c dysregulation in mice. Free Radic Biol Med 91, 224-235.

20. Riuzzi F, Sorci G, Sagheddu R, et al. (2012) HMGB1-RAGE regulates muscle satellite cell homeostasis through p38-MAPKand myogenin-dependent repression of Pax7 transcription. J Cell Sci 125, 1440-1454.

21. Miyazaki M \& Esser KA (2009) Cellular mechanisms regulating protein synthesis and skeletal muscle hypertrophy in animals. J Appl Physiol 106, 1367-1373.

22. Masiero E, Agatea L, Mammucari C, et al. (2009) Autophagy is required to maintain muscle mass. Cell Metab 10, 507-515.

23. Bonaldo P \& Sandri M (2013) Cellular and molecular mechanisms of muscle atrophy. Dis Model Mech 6, 25-39.

24. Hou X, Hu Z, Xu H, et al. (2014) Advanced glycation endproducts trigger autophagy in cadiomyocyte via RAGE/PI3K/ AKT/mTOR pathway. Cardiovasc Diabetol 13, 78 .

25. Ikeda K, Higashi T, Sano H, et al. (1996) N (epsilon)(carboxymethyl)lysine protein adduct is a major immunological epitope in proteins modified with advanced glycation end products of the Maillard reaction. Biochemistry 35 , 8075-8083.

26. Tan AL, Sourris KC, Harcourt BE, et al. (2010) Disparate effects on renal and oxidative parameters following RAGE deletion, AGE accumulation inhibition, or dietary AGE control in experimental diabetic nephropathy. Am J Physiol Renal Physiol 298, F763-F770.

27. Sourris KC, Harcourt BE, Penfold SA, et al. (2010) Modulation of the cellular expression of circulating advanced glycation end-product receptors in type 2 diabetic nephropathy. Exp Diabetes Res 2010, 974681.

28. Forbes JM, Cowan SP, Andrikopoulos S, et al. (2013) Glucose homeostasis can be differentially modulated by varying individual components of a western diet. J Nutr Biochem 24, 1251-1257.

29. Raymackers JM, Debaix H, Colson-Van Schoor $\mathrm{M}$, et al. (2003) Consequence of parvalbumin deficiency in the mdx mouse: histological, biochemical and mechanical phenotype of a new double mutant. Neuromuscul Disord 13, 376-387.

30. Toyoda T, Tanaka S, Ebihara K, et al. (2006) Low-intensity contraction activates the alpha1-isoform of 5'-AMP-activated protein kinase in rat skeletal muscle. Am J Physiol Endocrinol Metab 290, E583-E590.

31. Tsuda S, Egawa T, Kitani K, et al. (2015) Caffeine and contraction synergistically stimulate 5'-AMP-activated protein 
kinase and insulin-independent glucose transport in rat skeletal muscle. Physiol Rep 3, e12592.

32. Head SI, Greenaway B \& Chan S (2011) Incubating isolated mouse EDL muscles with creatine improves force production and twitch kinetics in fatigue due to reduction in ionic strength. PLOS ONE 6, e22742.

33. Yasuhara K, Ohno Y, Kojima A, et al. (2011) Absence of heat shock transcription factor 1 retards the regrowth of atrophied soleus muscle in mice. J Appl Physiol 111, 1142-1149.

34. Talmadge RJ \& Roy RR (1993) Electrophoretic separation of rat skeletal muscle myosin heavy-chain isoforms. J Appl Physiol 75, 2337-2340.

35. Ohno Y, Yamada S, Sugiura T, et al. (2011) Possible role of $\mathrm{NF}-\mathrm{kB}$ signals in heat stress-associated increase in protein content of cultured C2C12 cells. Cells Tissues Organs 194, 363-370.

36. Egawa T, Tsuda S, Ma X, et al. (2011) Caffeine modulates phosphorylation of insulin receptor substrate- 1 and impairs insulin signal transduction in rat skeletal muscle. J Appl Physiol 111, 1629-1636.

37. Egawa T, Goto A, Ohno Y, et al. (2015) Involvement of AMPK in regulating slow-twitch muscle atrophy during hindlimb unloading in mice. Am J Physiol Endocrinol Metab 309, E651-E662.

38. Watanabe H, Ogasawara M, Suzuki N, et al. (1992) Glycation of myofibrillar protein in aged rats and mice. Biosci Biotechnol Biochem 56, 1109-1112.

39. Katayama S, Haga Y \& Saeki H (2004) Loss of filamentforming ability of myosin by non-enzymatic glycosylation and its molecular mechanism. FEBS Lett 575, 9-13.

40. Syrovy I \& Hodny Z (1992) Non-enzymatic glycosylation of myosin: effects of diabetes and ageing. Gen Physiol Biophys 11, 301-307.

41. Avigad G, Kniep A \& Bailin G (1996) Reaction of rabbit skeletal myosin with D-glucose 6-phosphate. Biochem Mol Biol Int 40, 273-284.

42. Ramamurthy B, Hook P, Jones AD, et al. (2001) Changes in myosin structure and function in response to glycation. FASEB J 15, 2415-2422.

43. Snow LM, Fugere NA \& Thompson LV (2007) Advanced glycation end-product accumulation and associated protein modification in type II skeletal muscle with aging. J Gerontol A Biol Sci Med Sci 62, 1204-1210.

44. Pette D \& Staron RS (2000) Myosin isoforms, muscle fiber types, and transitions. Microsc Res Tech 50, 500-509.

45. Harridge SD, Bottinelli R, Canepari M, et al. (1996) Whole-muscle and single-fibre contractile properties and myosin heavy chain isoforms in humans. Pflugers Arch $\mathbf{4 3 2}$, 913-920.

46. Snow LM \& Thompson LV (2009) Influence of insulin and muscle fiber type in nepsilon-(carboxymethyl)-lysine accumulation in soleus muscle of rats with streptozotocin-induced diabetes mellitus. Pathobiology 76, 227-234.

47. Snow LM, Lynner CB, Nielsen EM, et al. (2006) Advanced glycation end product in diabetic rat skeletal muscle in vivo. Pathobiology 73, 244-251.

48. Ramamurthy B \& Larsson L (2013) Detection of an agingrelated increase in advanced glycation end products in fast- and slow-twitch skeletal muscles in the rat. Biogerontology $\mathbf{1 4}$ 293-301.

49. Seale P, Sabourin LA, Girgis-Gabardo A, et al. (2000) Pax7 is required for the specification of myogenic satellite cells. Cell 102, 777-786.

50. Hawke TJ \& Garry DJ (2001) Myogenic satellite cells: physiology to molecular biology. J Appl Physiol 91, 534-551.

51. Francetic $\mathrm{T} \& \mathrm{Li} \mathrm{Q}$ (2011) Skeletal myogenesis and Myf5 activation. Transcription 2, 109-114.

52. Perry RL \& Rudnick MA (2000) Molecular mechanisms regulating myogenic determination and differentiation. Front Biosci 5, D750-D767.

53. Andres V \& Walsh K (1996) Myogenin expression, cell cycle withdrawal, and phenotypic differentiation are temporally separable events that precede cell fusion upon myogenesis. J Cell Biol 132, 657-666.

54. Mak KL, To RQ, Kong Y, et al. (1992) The MRF4 activation domain is required to induce muscle-specific gene expression. Mol Cell Biol 12, 4334-4346.

55. Wheeler MT, Snyder EC, Patterson MN, et al. (1999) An E-box within the MHC IIB gene is bound by MyoD and is required for gene expression in fast muscle. Am J Physiol 276, C1069-C1078.

56. Hughes SM, Koishi K, Rudnicki M, et al. (1997) MyoD protein is differentially accumulated in fast and slow skeletal muscle fibres and required for normal fibre type balance in rodents. Mech Dev 61, 151-163.

57. Ekmark M, Rana ZA, Stewart G, et al. (2007) Dephosphorylation of MyoD is linking nerve-evoked activity to fast myosin heavy chain expression in rodent adult skeletal muscle. J Physiol 584, 637-650.

58. Macharia R, Otto A, Valasek P, et al. (2010) Neuromuscular junction morphology, fiber-type proportions, and satellite-cell proliferation rates are altered in $\mathrm{MyoD}(-/-)$ mice. Muscle Nerve 42, 38-52.

59. Caiozzo VJ, Wu YZ, Baker MJ, et al. (2004) Effects of denervation on cell cycle control in laryngeal muscle. Arch Otolaryngol Head Neck Surg 130, 1056-1068.

60. Vissing K, Andersen JL, Harridge SD, et al. (2005) Gene expression of myogenic factors and phenotype-specific markers in electrically stimulated muscle of paraplegics. J Appl Physiol 99, 164-172.

61. Coughlan MT, Yap FY, Tong DC, et al. (2011) Advanced glycation end products are direct modulators of beta-cell function. Diabetes 60, 2523-2532.

62. Peppa M, He C, Hattori M, et al. (2003) Fetal or neonatal low-glycotoxin environment prevents autoimmune diabetes in NOD mice. Diabetes 52, 1441-1448.

63. Peppa M, Brem H, Ehrlich P, et al. (2003) Adverse effects of dietary glycotoxins on wound healing in genetically diabetic mice. Diabetes 52, 2805-2813.

64. Diamanti-Kandarakis E, Piperi C, Korkolopoulou $\mathrm{P}$, et al. (2007) Accumulation of dietary glycotoxins in the reproductive system of normal female rats. J Mol Med 85, 1413-1420.

65. Patel R, Baker SS, Liu W, et al. (2012) Effect of dietary advanced glycation end products on mouse liver. PLOS ONE 7, e35143. 